\title{
Availability Analysis of Different PON Models
}

\author{
Katarina Radoš ${ }^{1}$ and Ivan Radoš ${ }^{2}$ \\ ${ }^{1}$ University of Split, Split, Croatia \\ ${ }^{2}$ HT d.o.o. Mostar, Mostar, Bosnia and Herzegovina
}

https://doi.org/10.26636/jtit.2017.116517

\begin{abstract}
Due to the increasing demands of individual users, Passive Optical Networks (PON) are a promising technology for future broadband access networks. Reliable access to network services is a very important feature, so the availability of the connection is becoming one of the most important requirements. Failure of the optical fiber occurring between the Optical Line Terminal (OLT) and the passive optical splitter can cause the services becoming unavailable for a large number of users, so it is necessary to prevent such an occurrence by providing backup resources - in this case a spare optical fiber. When constructing the spare path (protection fiber), it is important to keep in mind that, if possible, the working and the spare fiber should not be positioned within the same cable. Failure of the optical fiber between the passive splitter and the individual user also means a loss of service for that user. In that case, protection may be offered by adding spare fibers. The question is, however, whether such a solution is cost-effective, as it would lead to a significant cost increase in the construction of the access network. This paper presents the availability analysis conducted for different PON models.
\end{abstract}

Keywords—availability, failure, Passive Optical Network, protection fiber.

\section{Introduction}

The increasing number of Internet users and the growing bandwidth required by new applications, such as online games, telemedicine and distance learning, are factors that force operators to turn to new architectures. Future access networks must be capable of offering high bandwidth-peruser rates, with their capital and operating costs remaining at the lowest possible level. Therefore, optical access technologies are proposed as the best solution, with the Passive Optical Network (PON) being widely accepted as a promising technology for future broadband optical access networks that may be offered in various implementation scenarios, such as Fiber To The Home (FTTH) and Fiber To The Building (FTTB). One of the most important advantages of the PON network is that the Optical Distribution Network (ODN) consists of passive elements (optical fibers and passive optical splitters) only, which completely eliminates electromagnetic interference, improves reliability and availability of the system, and reduces the cost of maintenance [1]. In addition, the increasing importance of constant access to the Internet for people in everyday life leads to error management as an important challenge in future optical access networks. Individual users, despite requiring minimal interruptions, are unable to afford additional costs to improve reliability. So, operators should try to continue the provision of services in the case of a failures affecting the network, which requires additional costs associated with the deployment of various protection schemes. Availability-related requirements may nevertheless depend on the user's profile. Business users are looking for complete protection, covering end-to-end operations. Connection availability greater than $99.99 \%$ (4 nines) must be guaranteed, for example, for some business users, while most residential users may tolerate lower availability rates. However, costs incurred in order to offer additional protection layers may be significant, as such deployment involves doubling, resources such as fiber optics and optical line terminals. It was shown that the availability of 4 nines or more cannot be achieved without a protection path between the Central Office (CO) and end user. So, PON networks should be able to offer end-to-end support for some business users, when it is required [2].

This paper focuses on the implementation of the PON model, which will significantly improve the availability of the connection between the Optical Line Terminal (OLT) and end users. Of course, fiber protection extended from the optical splitter to the end user is not of the same importance as fiber protection between the OLT and the optical splitter, as an interruption between the OLT and the optical splitter will lead to a disconnection to all users that are connected to the OLT. At the same time, an interruption between the optical splitter and the Optical Network Unit (ONU) leads to a disconnection for that specific user only. Protection of any part of the network must be realized within $50 \mathrm{~ms}$, i.e. in accordance with the international standard. The present paper shows also the impact of the fiber length on the availability ratio.

The remaining parts of this paper are organized in the following manner. Section 2 describes the basic PON model. The general PON protection schemes are shown in Section 3. Section 4 describes availability in general. Section 5 shows PON protection and availability models, and Section 6 contains calculations and comments.

\section{Basic Model of Passive Optical Network}

The basic PON model consists of the following elements: OLT, optical splitter and ONU. 


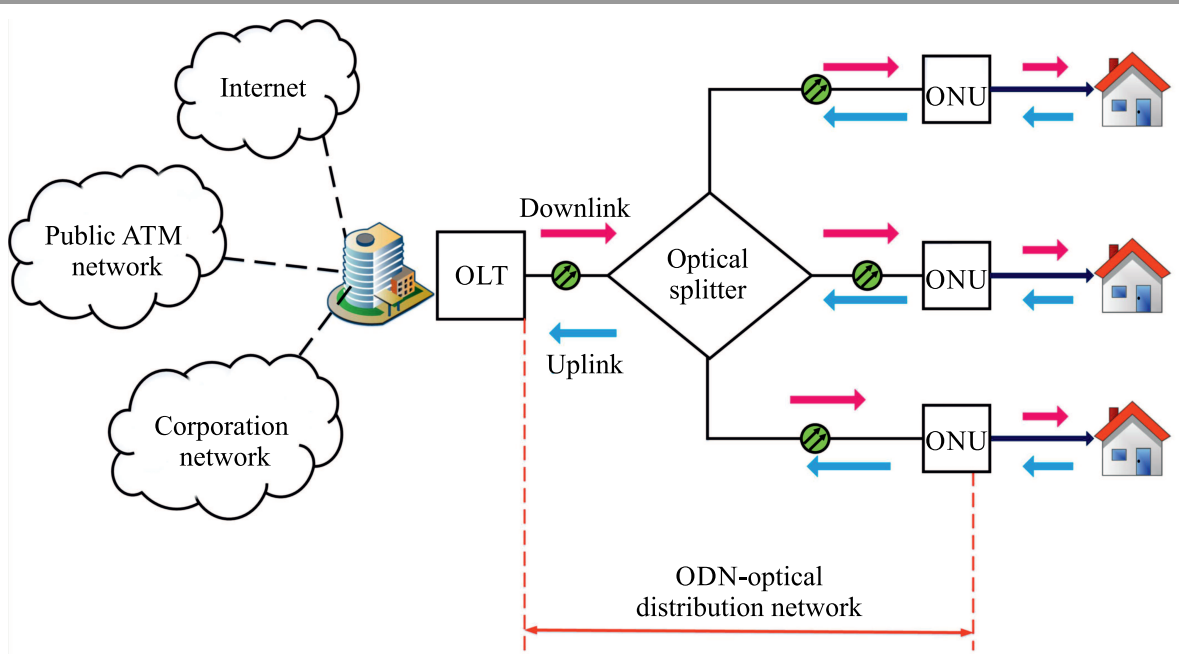

Fig. 1. The basic model of a passive optical network.

Figure 1 shows the basic PON model. Data transfer is always performed between the OLT and different ONUs via an optical splitter that performs multiplexing and demultiplexing of signals. OLT and ONU are active parts of the PON network, because they perform the electro-optical and opto-electrical signal conversion.

The OLT is located in the $\mathrm{CO}$ of the operator. It represents the interface between the public network and access network and also controls bidirectional flow of information via the ODN.

The OLT must be able to support transmissions over a distance of $20 \mathrm{~km}$. The role of the OLT, in the downlink direction (from OLT to users), is to transmit data, voice and video form the public network, via a single-mode optical fiber, to ONUs. The OLT performs also the electro-optical conversion in the downlink direction [1], [2].

In the uplink direction (from users to OLT), in turn, the OLT accepts, performs opto-electrical conversion and distributes traffic from the users. Simultaneous transmission of specific types of services via the same optical fiber in the ODN is enabled by using different wavelengths for each direction. For downlink transmissions, PON uses the wavelength of $1490 \mathrm{~nm}$ for a combined voice and data traffic, and the wavelength of $1550 \mathrm{~nm}$ for video content. As far as uplink is concerned, PON uses the wavelength of $1310 \mathrm{~nm}$ for voice and data traffic. Video services are disabled in the uplink direction, because there are no video services which would send video data in this direction [2], [3].

The optical splitter is a passive bidirectional component with one input and multiple outputs. At the entrance of the splitter, the optical power of signals that are sent to the end users is divided evenly at the ratio of $1: \mathrm{N}$ (commonly 1:32), where $\mathrm{N}$ is the number of end users connected to the optical splitter. Optical splitter requires no power supply and is maintenance-free.

ONUs are located at the user's premises and they serve as an interface with the end users. ONUs are connected via to the OLT via an optical splitter. The ONU performs opto- electrical conversion in the downlink direction [3], electro-optical conversion in the uplink direction, and packet filtering based on the destination address in the packet's header. The ODN consists of passive optical splitters and optical fibers. ODN consists of passive optical splitters and optical fibers.

\section{General PON Protection Schemes}

There are many different protection approaches, but they are confined, in principle, to the protection of the optical fiber (cable) and hardware used in optical access networks. Four types of protection are described in [4] and are based on the ITU-T Recommendation G.984.1, as shown in Fig. 2. Figure $2 \mathrm{a}$ shows the first type of protection in which the protection (spare) fiber is installed between the OLT and the optical splitter (type 1:N). No switching protocol is required for the OLT/ONU in Fig. 2a, since switching is only applied for optical fibers. The optical switch is located over the working and the protection fiber connected to the 1:N optical splitter.

Under normal conditions, the optical switch is in the bar state and traffic is carried over the working fiber. In case of a failure affecting the working fiber, the optical switch detects the loss of optical signal, passes into the cross state and redirects the traffic onto the protection fiber.

The second type of protection is shown in Fig. 2b. In that scenario, a spare OLT and a spare optical fiber are installed (marked with a dashed, bold, red line). No switching protocol is required since switching is carried out in the OLT only. The spare OLT is in the standby mode. In the case of a failure affecting the working fiber, the optical switch redirects traffic from the working OLT to the protection fiber. If the optical switch detects a loss of signal from the working OLT, it performs switching and redirects traffic from the spare OLT to the working fiber. Fiber interruption between the optical splitter and each ONU will not cause any 
(a)

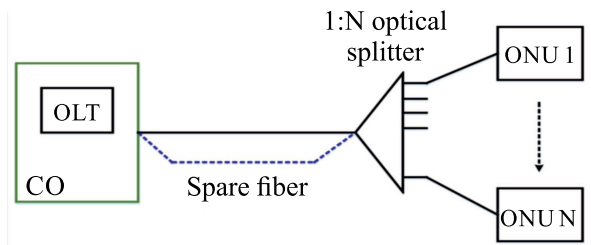

(c)

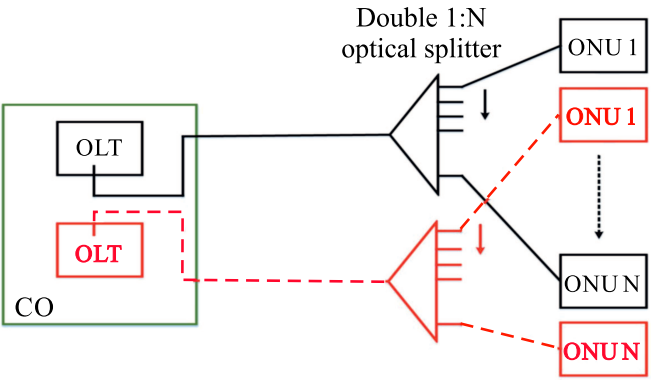

(b)

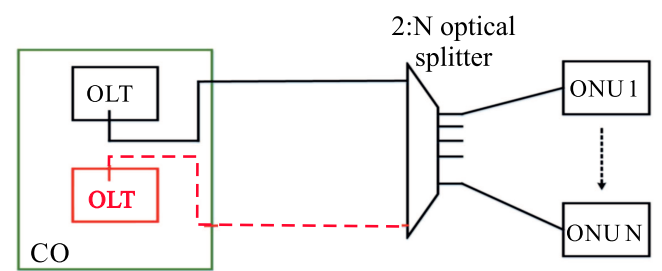

(d)

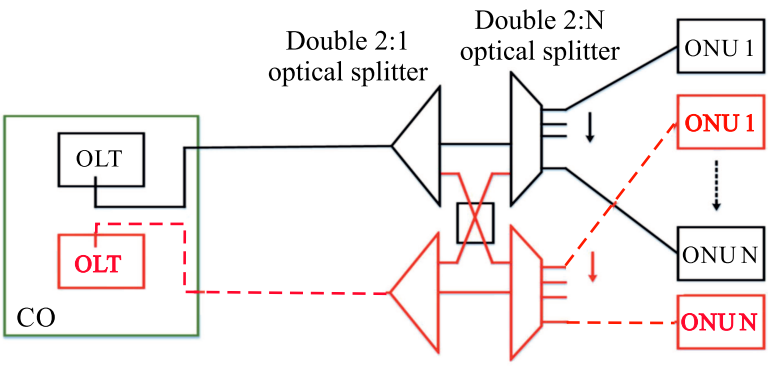

Fig. 2. General protection schemes by ITU-T. (For color pictures, see www.nit.eu/publications/journal-jtit)

switch reaction on the OLT side, so the concerned ONU affected by this kind of optical fiber interruption will not be protected [4].

Figure 2c shows the third protection scheme in which the OLT and ONU the are equipped with redundant modules. For this type of protection, 1:N optical splitters are needed. In this case the spare PON circuit that includes the OLT and the ONU can be activated in the case of a failure on the OLT or ONU side. To activate the spare PON circuit, constant synchronization between working and spare modules is required. With this type of protection, the network is able to survive all individual failures.

A network with complete redundancy, offering protection of equipment and optical fibers, is shown in Fig. 2d. This architecture also uses two 2:N passive optical splitters that connect $\mathrm{N}$ users with the geographically separated optical fibers. An additional optical switch with the controlling module is embedded in each ONU. This architecture can survive a failure of the OLT, the ONU's receiver and the passive splitter. It also can survive optical fiber failures between the OLT and the passive splitter, as well as between the passive splitter and the individual ONUs. Being the most reliable architecture, it is also the most expensive variant that requires highly complex management.

\section{Availability in General}

Availability $A$ is often used to describe the performance of a system, and is defined as the probability of the system's proper operation at some point in time $t$, under condition that system was working properly at time $t=0$, that it can enter fault states (maintenance or failure) which are always fixed and followed by the system's return to a working state [5]. The availability of a system within a period of time is also defined as the ratio between time in which the system operates correctly, and the total time elapsed. If the average time to failure (Mean Time To Failure - MTTF) and the average time to repair (Mean Time To Repair MTTR) are known, availability can be calculated using the following formula:

$$
A=\frac{\mathrm{MTTF}}{\mathrm{MTTF}+\mathrm{MTTR}} .
$$

Usually, MTTF is not known, so the term in the denominator is defined as the average time between failures (MTBF - Mean Time Between Failures) [5] and the availability can be written as:

$$
A=\frac{\mathrm{MTTF}}{\mathrm{MTTB}} \text {. }
$$

Unavailability $U$ is the probability that is complementary to availability, i.e. $U=1-A[6]$, meaning that it is the probability of the system's failure to work properly at some point in time:

$$
U=\frac{\mathrm{MTTR}}{\mathrm{MTTF}+\mathrm{MTTR}} \cong \lambda \times \mathrm{MTTR},
$$

where $\lambda$ is the intensity of failure that is expressed in FIT, $1 \mathrm{FIT}=1$ failure $/ 10^{9} \mathrm{~h}$.

When reporting on system performance, unavailability is often expressed as the average time of failure (Mean Down Time - MDT) [6]:

$$
\mathrm{MDT}=365 \times 24 \times 60 \times U[\mathrm{~min} / \text { year }] .
$$

\subsection{Basic Structures of Availability}

There are two basic structures of availability: serial (nonredundant) and parallel (redundant) [7]. In the serial structure, the failure of any element of the system equals the failure of the entire system (the system will work only if 
each element of the system is operating correctly). Serial structure of a system is shown in Fig. 3.

In light of the above, a path which consists of $\mathrm{N}$ elements is correct if each element is in the "correct" state, which may be represented by the following logical equation:

$$
\mathrm{P}_{s, \mathrm{~N}}=\mathrm{x}_{1} \cap \mathrm{x}_{2} \cap \ldots \cap \mathrm{x}_{\mathrm{N}},
$$

where $\mathrm{P}_{s, \mathrm{~N}}$ is the probability of the proper operation of a serial structure of $\mathrm{N}$ elements, $\mathrm{x}_{1}, \mathrm{x}_{2}, \ldots, \mathrm{x}_{\mathrm{N}}$ is the proper operation of each element. The path availability is expressed, in that case, by:

$$
A\left(\mathrm{P}_{s, \mathrm{~N}}\right)=p\left(\mathrm{x}_{1} \cap \mathrm{x}_{2} \cap \ldots \cap \mathrm{x}_{\mathrm{N}}\right) .
$$

If it is assumed that the failures of elements are independent, i.e., the failure of one element does not cause the failure of other elements, availability can be calculated in the following manner:

$$
\begin{aligned}
A\left(\mathrm{P}_{s, \mathrm{~N}}\right) & =p\left(\mathrm{x}_{1}\right) \times p\left(\mathrm{x}_{2}\right) \times \cdots \times p\left(\mathrm{x}_{N}\right)= \\
& =\mathrm{A}_{x_{1}} \times \mathrm{A}_{x_{2}} \times \cdots \times \mathrm{A}_{x_{\mathrm{N}}} .
\end{aligned}
$$

Figure 4 shows the parallel system structure in which the failure of any element does not mean an interruption in communication (the system will work even if some of the elements are defective).

In most cases the structure comprises two elements, so the probability of proper operation of such a structure can be represented by [7]:

$$
\mathrm{P}_{p, 2}=\mathrm{x}_{1} \cup \mathrm{x}_{2} \text {. }
$$

In that case, path availability is

$$
A\left(\mathrm{P}_{p, 2}\right)=p\left(\mathrm{x}_{1} \cup \mathrm{x}_{2}\right) .
$$

Assuming that the failures of elements are mutually independent, availability can be calculated as follows:

$$
\begin{aligned}
A\left(\mathrm{P}_{p, 2}\right) & =p\left(\mathrm{x}_{1}\right)+p\left(\mathrm{x}_{2}\right)-p\left(\mathrm{x}_{1}\right) \times p\left(\mathrm{x}_{2}\right)= \\
& =\mathrm{A}_{x_{1}}+\mathrm{A}_{x_{2}}-\mathrm{A}_{x_{1}} \times \mathrm{A}_{x_{2}} .
\end{aligned}
$$

\section{PON Protection and Availability Models}

In this study the standard PON models (i.e., the basic model and the protection model) defined by ITU-T are considered, and serve as a basis for availability calculations [4], [8], [10]. New models that are upgraded based on the ITU-T models are described as well. Models defined by ITU-T are shown in Figs. 5-8, and models worked out based on the ITU-T models are presented in Figs. 9-10. Distance

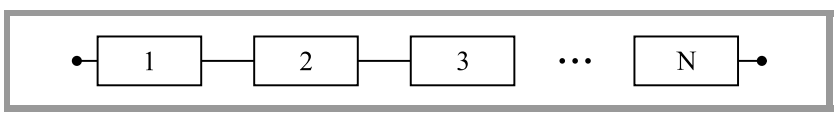

Fig. 3. Appearance of the serial system structure.

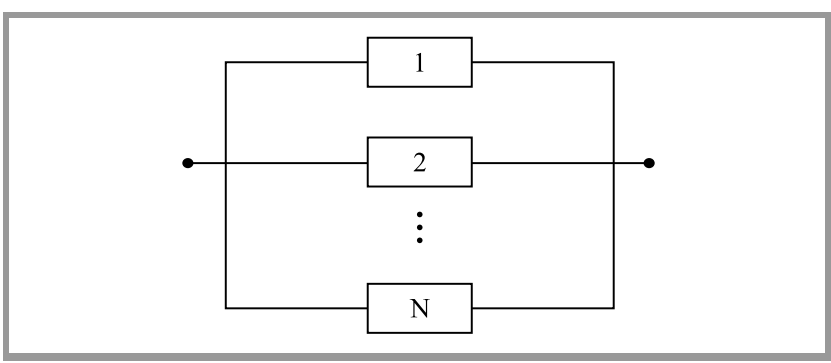

Fig. 4. Appearance of the parallel system structure.

between the OLT and the ONU equals, in all models, 20 $\mathrm{km}$.

Figure 5 shows the basic PON model without protection, which consists of the OLT, FF (feeder fiber), the 1:N splitter, DF (distribution fiber) and the ONU.

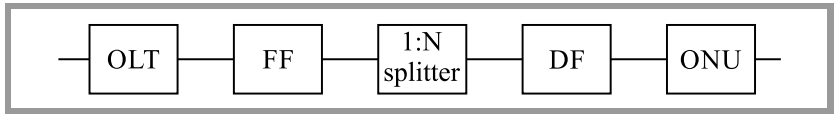

Fig. 5. Basic PON model.

The PON model with feeder fiber protection (one FF block is the working component and the other FF block the is protection component) is shown in Fig. 6. The system will function if there is at least one path that connects the start to the end and does not pass through a failed component.

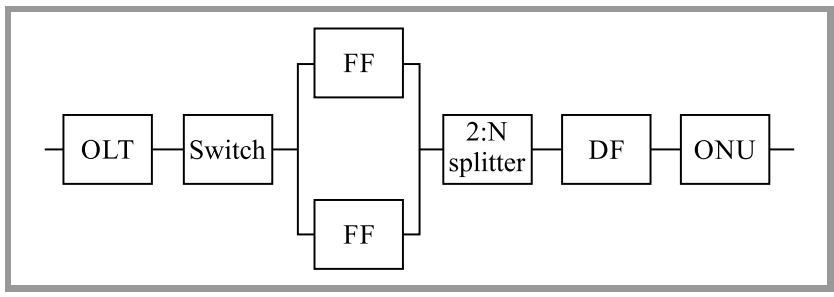

Fig. 6. Type 1 - based on scheme shown in Fig. 2a.

Figure 7 shows a redundant PON model which consists of two parallel structures. The first structure is made of the OLT, FF and the 1:2 splitter, and the other structure consists of the 2:N splitter, DF, and the ONU.

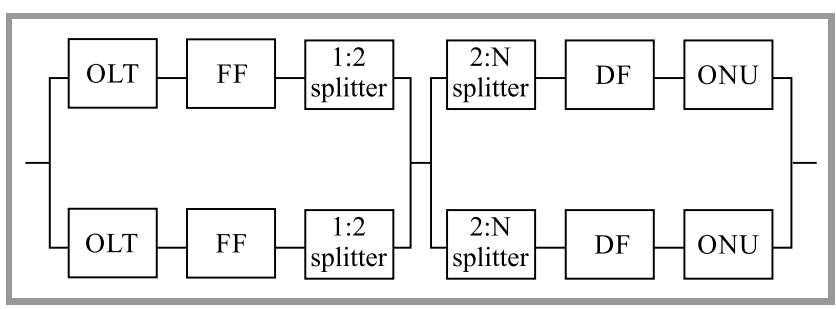

Fig. 7. Type 2 - based on Fig. 2 d.

The PON model with a redundant OLT, FF and 1:2 splitter is shown in Fig. 8.

Based on previous 4 models, two new models that involve optical fiber protection leading to individual users (DF), 


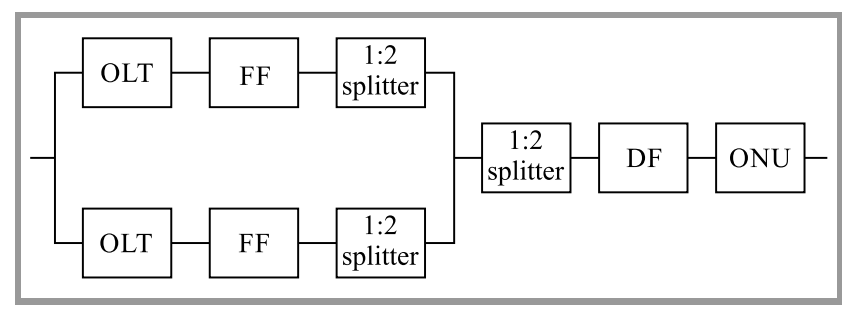

Fig. 8. Type 3 - based on Fig. 2 b.

or ONU protection, are introduced. The reason for which the new models have been developed is that they are still concerned with the individual user, so the question remains whether it is profitable to install the protection as shown in type 2. The key components (OLT, FF, 1:2, 2:N) are protected because the failure of any of them disables the provision of the service to all users (ONUs). The model shown in Fig. 9 consists of a parallel structure made up of the OLT, FF and the 1:2 splitter, as well as a parallel structure made of the $2: \mathrm{N}$ splitter, DF, and the ONU.

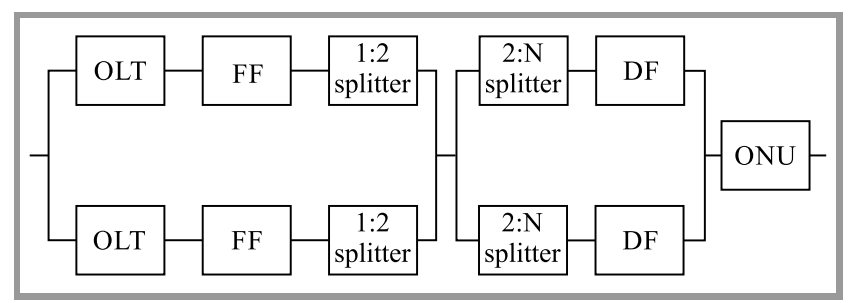

Fig. 9. Type 4.

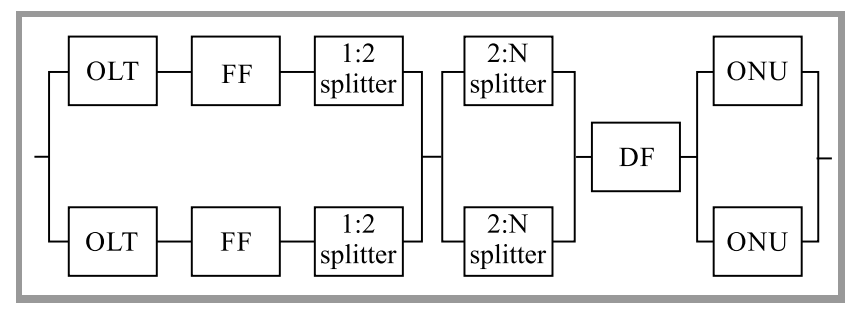

Fig. 10. Type 5.

Figure 10 shows the PON model in which the OLT, FF and the 1:2 splitter make up a parallel structure. The $2: \mathrm{N}$ splitter and the ONU are redundant and separated.

\section{Calculations and Comments}

In this paper three FTTH deployment cases are analyzed [8]:

- Low customer density case for sparsely populated areas. In this case, FF is $15 \mathrm{~km}$ and DF is $5 \mathrm{~km}$ long;

- High customer density case for densely populated areas. FF is $18 \mathrm{~km}$ and DF is $2 \mathrm{~km}$ long;

- Extra high customer density case for very densely populated areas (large apartment blocks, e.g. in China, Russia, Poland, etc.). FF is $19.7 \mathrm{~km}$ and DF is $0.3 \mathrm{~km}$ long.
Firstly, the low customer density case will be explained. As already mentioned, FF is $15 \mathrm{~km}$ and DF is $5 \mathrm{~km}$ long in this case. Table 2 shows low customer density case for PON models. The parameters that are calculated for each model include total unavailability, total availability and total MDT. Unavailability rates for each component in the PON network are shown in Table 1 and are taken from [8], where $\mathrm{N}$ represents the number of ONUs.

Table 1

Unavailability of the components

\begin{tabular}{|c|c|}
\hline Component & Unavailability \\
\hline \hline OLT (TDM PON) & $5.12 \cdot 10^{-7}$ \\
\hline ONU (TDM PON) & $1.54 \cdot 10^{-6}$ \\
\hline $1: 2(2: 2)$ splitter & $3.00 \cdot 10^{-7}$ \\
\hline $1: \mathrm{N}(2: \mathrm{N})$ splitter & $7.20 \cdot 10^{-7}$ \\
\hline Fiber [per km] & $1.37 \cdot 10^{-5}$ \\
\hline Switch & $1.20 \cdot 10^{-6}$ \\
\hline
\end{tabular}

Before commenting on the results of calculations, it is important to say that the serial availability structure is calculated, for all models, according to Eqs. (7) and (10), where Eq. (7) is related to the serial structure, and Eq. (10) is related to the parallel structure.

Total unavailability for all models is obtained from the expression $U=1-A$. Furthermore, total MDT is calculated with the use of Eq. (4). The basic PON model is the ITU-T model without protection and represents the serial availability structure.

As shown in Table 2, total MDT for the basic model is 145.463 minutes per year. Model type 1 has FF protection which represents the parallel structure of availability that is calculated according to Eq. (10). In the case of failure of the working FF, all traffic is switched to the protection FF. Total MDT is better than in the case of the basic model and equals $38.113 \mathrm{~min} /$ year.

In type 2, all components of the network are redundant and it is a very well protected network, which MDT being very low (0.024 min/year). As shown in Table 2, MDT for type 2 is 5832 times lower than for the basic model, and 1528 times lower than for type 1 .

Type 3 has three redundant components: the OLT, FF and the 1:2 splitter. This network is unavailable for $37.213 \mathrm{~min} /$ year. Better availability would be achieved if there was a redundant ONU, but ONU protection is not so important because if interruption of the optical fiber occurs between the OLT and the optical splitter, both the working and the redundant ONU will be deprived of the signal.

All components in type 4 are redundant except ONU. This model is very well protected with small MDT (0.834 $\mathrm{min} /$ year).

Type 5 has all redundant components except for DF, and, consequently, has a higher unavailability rate.

For the high customer density case, FF is $18 \mathrm{~km}$ and DF is $2 \mathrm{~km}$, as already mentioned. Table 3 shows the values 
Table 2

Low customer density case

\begin{tabular}{|l|c|c|c|}
\hline Model & Total availability & Total unavailability & Total MDT [min/year] \\
\hline \hline Basic PON & 0.999723243 & $2.76 \cdot 10^{-4}$ & 145.463 \\
\hline Type 1 & 0.999927486 & $7.25 \cdot 10^{-5}$ & 38.113 \\
\hline Type 2 & 0.999999953 & $4.74 \cdot 10^{-8}$ & 0.024 \\
\hline Type 3 & 0.999929198 & $7.08 \cdot 10^{-5}$ & 37.213 \\
\hline Type 4 & 0.999998413 & $1.58 \cdot 10^{-6}$ & 0.834 \\
\hline Type 5 & 0.999931457 & $6.85 \cdot 10^{-5}$ & 36.025 \\
\hline
\end{tabular}

Table 3

High customer density case for densely populated areas

\begin{tabular}{|l|c|c|c|}
\hline Model & Total availability & Total unavailability & Total MDT [min/year] \\
\hline \hline Basic PON & 0.999723236 & $2.76 \cdot 10^{-4}$ & 145.467 \\
\hline Type 1 & 0.999968567 & $3.14 \cdot 10^{-5}$ & 16.521 \\
\hline Type 2 & 0.999999938 & $6.19 \cdot 10^{-8}$ & 15.621 \\
\hline Type 3 & 0.999970279 & $2.97 \cdot 10^{-5}$ & 0.842 \\
\hline Type 4 & 0.999998398 & $1.60 \cdot 10^{-6}$ & 14.433 \\
\hline Type 5 & 0.999972539 & $2.74 \cdot 10^{-5}$ & \\
\hline
\end{tabular}

Table 4

Extra high customer density case for very densely populated areas

\begin{tabular}{|l|c|c|c|}
\hline Model & Total availability & Total unavailability & Total MDT [min/year] \\
\hline \hline Basic PON & 0.99972323 & $2.76 \cdot 10^{-4}$ & 145.470 \\
\hline Type 1 & 0.999991845 & $8.15 \cdot 10^{-6}$ & 4.286 \\
\hline Type 2 & 0.999999927 & $7.31 \cdot 10^{-8}$ & 0.038 \\
\hline Type 3 & 0.999993557 & $6.44 \cdot 10^{-6}$ & 3.386 \\
\hline Type 4 & 0.999998387 & $1.61 \cdot 10^{-6}$ & 0.847 \\
\hline Type 5 & 0.999995817 & $4.18 \cdot 10^{-6}$ & 2.198 \\
\hline
\end{tabular}

of total availability, total unavailability and total MDT for the high customer density case considered for each of the models. As one can see, total MDT for the basic PON is almost equal to that experienced in the low customer density case (see Table 2).

Total MDT for type 1 is 2.3 times lower than in the low customer density case $(\mathrm{FF}=15 \mathrm{~km})$, because the longer fiber $(\mathrm{FF}=18 \mathrm{~km})$ is protected in the high customer density case. Total MDT decreases because the unprotected distribution fiber is shorter and its unavailability is correspondingly lower. In type 2 , total MDT in the high customer density case (DF $=2 \mathrm{~km}$ ) is a little higher than in the low customer density case $(\mathrm{DF}=5 \mathrm{~km})$, because the shorter fiber is protected. Total MDT increases because the unprotected distribution fiber is longer.

Type 3 has approximately the same availability as type 1 .

For type 4 the same is valid as for type 2 (the protection of the shorter fiber renders a higher total MDT, meaning that the network has a higher unavailability rate per year). For type 5 availability is approximately the same as for type 3 .
Based on all the calculations presented above, one may conclude that type 2 is the best network model in both the low customer density and the high customer density scenarios, because all components are protected. Therefore, it has the lowest MDT, meaning that the network is unavailable for only 0.024 minutes per year in the low customer density case. Despite the best availability ratio, type 2 is expensive to construct and maintain, because all of its components are redundant. Type 4 has very good availability, although it is lower than in type 2. As far as cost-effectiveness is concerned, type 4 is cheaper than type 2 because the ONU is not redundant.

Compared to type 2 and type 4 models, type 5 has a worse availability ratio, but it is the cheapest because no spare fiber is necessary to protect the ONU.

As expected, basic PON model with serial structure has the worst availability because there are no redundant components.

Table 4 shows the values of total availability, total unavailability and total MDT for the extra high customer density 
case of each model. In this case, results for basic PON, type 2 and type 4, are roughly equal to those in the second case (Table 3). Total MDT for type 1, type 3 and type 5 is lower than in the second case because the fiber protected is longer $(\mathrm{FF}=19.7 \mathrm{~km})$. In this case, DF protection makes no sense because we are dealing with a very densely populated area and relatively short distances $(300 \mathrm{~m})$, so the probability of failures is rather low.

\section{Conclusions}

This paper analyzes the availability of different PON models. Two deployment cases are shown, and it can be concluded, based on those cases, that type 2 , with all redundant components, is the model characterized by the best availability and the lowest MDT. This model is also the most expensive scenario, as all its components are redundant. As mentioned above, ONU protection is not so important, because if interruption of the optical fiber occurs between the OLT and the optical splitter, both the working and the redundant ONU will be deprived of the signal. Type 4 does not have ONU protection but still offers very good availability. Type 5, which is considerably cheaper than models 2 and 4, offers availability that is satisfactory for individual users and is also a promising solution for the construction of PON networks.

It can be also concluded that a network in which the longer fiber is protected offers better availability (see type 5 in Tables 2 and 3). Therefore, one may state that if the length of the fiber increases, MDT decreases and vice versa. It is very difficult to obtain a model characterized by very good availability and cost-effectiveness.

\section{References}

[1] C. Lam, Passive Optical Networks: Principles and Practice. London: Elsevier, 2008

[2] J. S. Asensi Pla, "Design of passive optical network", Master thesis, Brno University of Technology, May 2011.

[3] M. Kapov and J. Lorincz, Local and Access Networks, Split: FESB, 2013.

[4] ITU-T Recommendations: G983.1, 1998, and G984.1, 2003.

[5] L. Schwartz, D. Trstensky, and G. Cepciansky, "Reliability of telecommunications systems", University of Zilina, Slovakia, 2010, p. 193 (in Slovak).

[6] D. R. Shier, Network Reliability and Algebraic Structures. New York: Oxford University Press, 1991.

[7] I. Radoš, "Availability of self-healing ring networks based on wavelength division multiplexing", Ph.D. thesis, University of Split, 2012.

[8] L. Wosinska, J. Chen, and C. P. Larsen, "Fiber access networks: Reliability analysis and Swedish broadband market", IEICE Trans. Commun., vol. E92-B, no. 10, pp. 3006-3013, 2009.
[9] W. A. Imtiaz, M. Waqas, P. Mehar, and Y. Khan, "Self-healing hybrid protection architecture for passive optical networks", Int. J. of Adv. Comp. Sci. and Appl. (IJACSA), vol. 6, no. 8, 2015 (doi: 10.14569/IJCSA.2015.060819).

[10] ITU-T G-series Recommendations - Supplement 51, 2016.

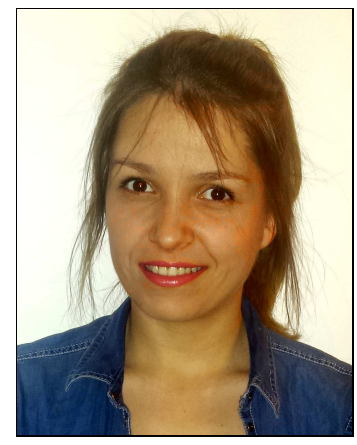

Katarina Radoš received her M.Sc. in Communication and Information Technology from the University of Split, Faculty of Electrical Engineering, Mechanical Engineering and Naval Architecture (2016). Currently, she is working at the Faculty of Electrical Engineering, Mechanical Engineering and Naval Architecture, Split, Croatia. Her scientific research focuses on optical communications, optical access networks (PON), reliability and availability of optical networks.

E-mail: krados@fesb.hr

University of Split

Faculty of Electrical Engineering, Mechanical Engineering and Naval Architecture

Kroz Smrdečac 47

21000 Split, Croatia

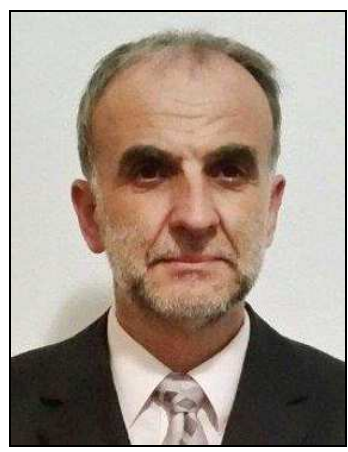

Ivan Radoš received the B.Sc. degree in Electrical Engineering from the University of Split, in 1983, M.Sc. degree from the University of Zagreb, in 2000 and Ph.D. from the University of Split, Croatia in 2012. In 1985 he joined the PTT (Post and Telecommunication) Tomislavgrad. Since 1992 he works at Department of Transmission Systems of the HT Mostar (Croatian Telecommunication). His research interests include: digital transmission systems, optical systems and networks, availability and reliability of telecommunication systems. He has published 10 papers in international conference proceedings, 5 papers in domestic journals and 8 papers in international journals. E-mail: ivan.rados@hteronet.ba

HT d.o.o. Mostar

Kneza Branimira bb

88000 Mostar, Bosnia and Herzegovina 